Document downloaded from:

http://hdl.handle.net/10251/49589

This paper must be cited as:

Guardiola García, C.; Plá Moreno, B.; Onori, S.; Rizzoni, G. (2014). Insight into the $\mathrm{HEV} / \mathrm{PHEV}$ optimal control solution based on a new tuning method. Control Engineering Practice. 29:247-256. doi:10.1016/j.conengprac.2014.01.022.

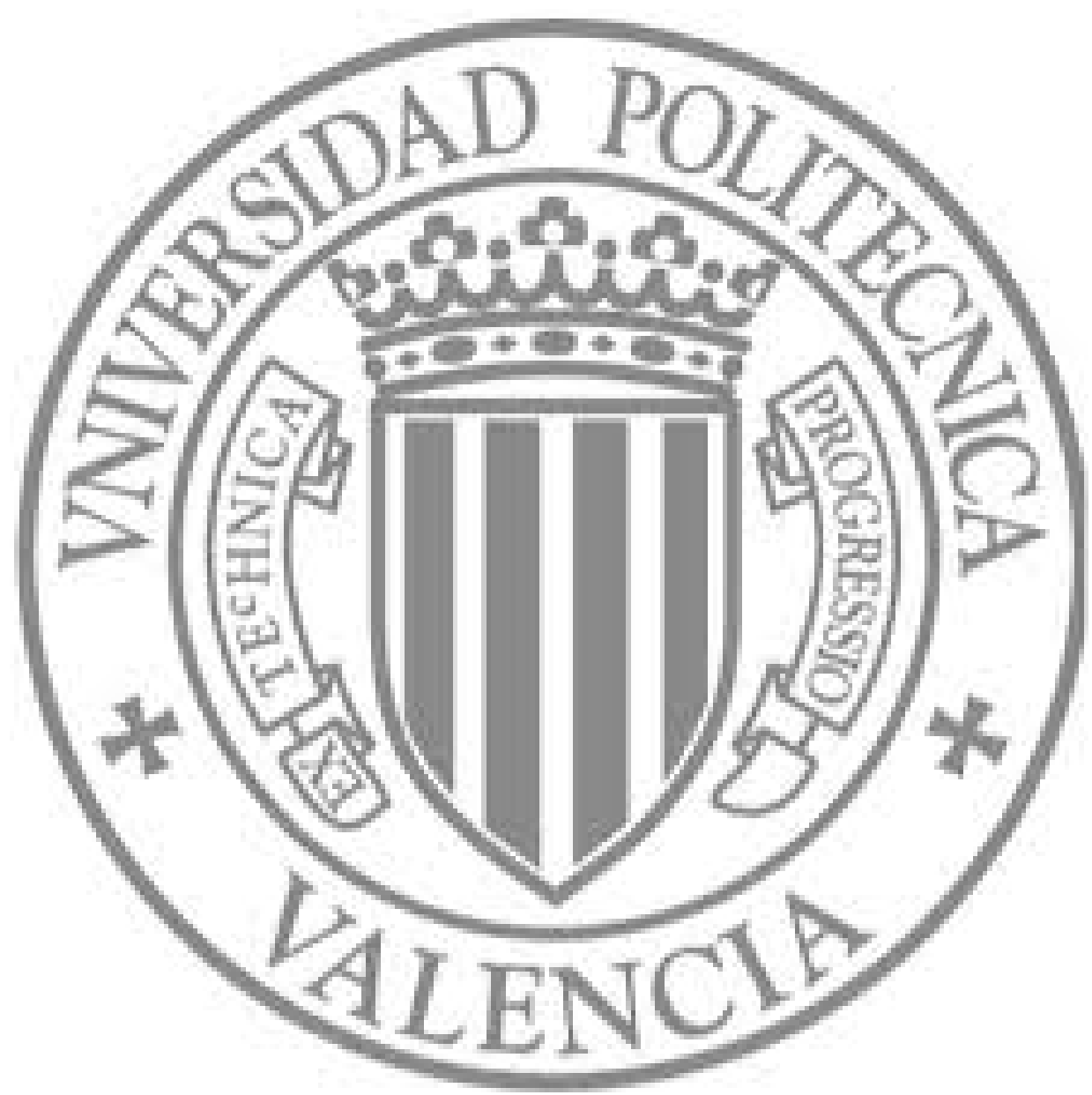

The final publication is available at

http://dx.doi.org/10.1016/j.conengprac.2014.01.022

Copyright Elsevier 


\title{
Insight into the HEV/PHEV optimal control solution based on a new tuning method
}

\author{
C. Guardiola ${ }^{\mathrm{a}}$, B. Pla ${ }^{\mathrm{a}}$, S. Onori ${ }^{\mathrm{b}}$, G. Rizzoni ${ }^{\mathrm{c}}$ \\ ${ }^{a}$ CMT-Motores Térmicos, Universitat Politècnica de València, Valencia, Spain. carguaga@mot.upv.es. Fax: +34963877659 \\ ${ }^{b}$ International Center for Automotive Research, Clemson University, Clemson,SC \\ ${ }^{c}$ Center of Automotive Research, Ohio State University, Columbus, $\mathrm{OH}$
}

\begin{abstract}
The paper presents a formulation of the energy management problem for Hybrid Electrical Vehicles and Plug-in Hybrid Electrical Vehicles alike, which permits to consider different cost indexes like fuel consumption, total and primary energy consumption, economic cost or $\mathrm{CO}_{2}$ footprint. In-depth analysis of the problem optimal solution is done by means of the application of the $\lambda$-plot method, which also permits the optimal tuning of other implementable control strategies. Such an approach is used to understand the effect of the selected cost index, the regional energetic share, the driving conditions, and for deriving rules for battery sizing.
\end{abstract}

Keywords: HEV, PHEV, hybrid electrical vehicle, range extender, energy management, optimal control, optimisation, automotive engine

\section{Introduction}

Increasing concern about global warming and availability of fossil fuel resources is pushing society to search for new solutions towards sustainable mobility (Mierlo et al., 2006). Accordingly, over the past decades important efforts started being carried out in order to increase the efficiency of internal combustion engines (ICE). Despite ground vehicles have been powered almost exclusively by ICEs since more than 100 years ago, alternative powertrain architectures are currently under evaluation for reducing transport ecological footprint (Fernández et al., 2011; Katrašnik, 2007a,b). Hybrid Electric Vehicles (HEV) combine a fuel-driven power source with an electric drivetrain allowing the engine to stop at idling and low load conditions where its efficiency is low (Xiong et al., 2009; Banjac et al., 2009). Regenerative braking is also possible with HEVs. Then, HEVs have aroused the interest of the transportation sector, at least as a temporary solution on the way to zero emission road vehicles.

$\mathrm{HEV}$ is a general term referring a vehicle whose powertrain combines an ICE with one or more electric machines and a reversible energy storage system, however its use is usually restricted to charge sustaining HEVs, i.e. the class of vehicles for which energy consumed comes ultimately from the fuel tank. On the other hand, Plug-in Hybrid Electric Vehicles (PHEVs) are able to recharge their batteries through direct connection to the energy grid, allowing depleting the battery during driving mission and then improving the vehicle fuel economy.

HEV control has been widely studied and many works address it through the optimal control theory (Sciarreta and Guzzella, 2007): optimal control policy is that providing the minimum fuel consumption while satisfying the battery

${ }^{1}$ C. Guardiola and B. Pla research has been partially supported by Ministerio de Ciencia e Innovación through Project TRA2010-16205 uDiesel, and the Universidad Politécnica de Valencia through grants PAID-00-11 2105 and PAID-00-11 2106. 
charge sustainability (Delprat et al., 2001). Nevertheless, the introduction of other primary energy sources than the fuel tank in the PHEV may require to take into account other costs in addition to fuel consumption, since battery recharging contribute to vehicle operation cost and emissions.

The present paper uses a general formulation of the energy management problem for HEV and PHEV alike. The formulation is able to easily consider different cost indexes (i.e. fuel consumption, total energy consumption, primary energy consumption, economic cost or $\mathrm{CO}_{2}$ emission footprint). The general problem is solved through three of the most common methods in HEV/PHEV energy management: Dynamic Programing (DP) (Mosbech, 1980; Sundström et al., 2010), Pontryagin Minimum Principle (PMP) (Serrao et al., 2011) and Equivalent Consumption Minimization (ECMS) (Paganelli et al., 2001; Won and Langari, 2005; Sciarretta et al., 2004).

The method used in this paper to tune both PMP and ECMS builds upon the results presented in Guardiola et al. (2012). The method is based on the numerical analysis of the optimal solution from DP and provides a suitable framework for comparing different control strategies. Such an approach will be used for highlighting the differences between the HEV and PHEV optimisation problem: while in the HEV control the final state of the battery is always constrained, in the case of the PHEV this constraint will only be active depending on the selected cost index, the energetic share and electrical grid characteristics, the driving conditions, and the battery size.

Although the methodologies shown in the paper are general and could be applied to a wide variety of powertrain configurations, the simulations have been restricted to the series hybrid configuration, which is being actively screened for PHEV (using the ICE engine as range extender).

\section{Problem formulation}

The Energy Management Problem in a HEV consists in finding the sequence of power split that minimises a cost index over a driving cycle. The index or function to minimise depends on the vehicle objectives and its application. Energy, economical cost and $\mathrm{CO}_{2}$ emissions are some of the most widely used variables to minimise and, for the sake of generality, the problem can be formally defined as finding the control law $u(t)$ over time $t$ that minimises the cost:

$$
J=\int_{t_{0}}^{t_{f}} P_{f}(u(t), t)+\alpha P_{b}\left(u(t), E_{b}(t), t\right) \mathrm{dt}
$$

where $P_{f}$ and $P_{b}$ are the fuel and battery power consumed at each instant of the driving cycle, and $\alpha$ is a weighting constant whose value depends on the optimisation objective. The battery power is considered positive when the battery is being discharged and negative when the battery is being charged. $E_{b}$ is the only state variable in the problem and represents the energy stored in the battery, whose dynamics is given by:

$$
\dot{E}_{b}=-P_{b}
$$

Although $E_{b}$ has been selected in the current formulation as state variable, all plotted results will be normalised by the total battery energy $E_{b, \max }$ resulting in the battery state of energy $S O E$ :

$$
S O E=\frac{E_{b}}{E_{b, \text { max }}}
$$

Both the $S O E$ or the battery state of charge $S O C$ are other possible choices for the state variable; this aspect will be treated in the Appendix.

Note that in the case of a HEV, it is, a charge-sustaining hybrid vehicle, the battery may not be externally recharged. Usual assumption (Delprat et al., 2001; Ambühl et al., 2010; Stockar et al., 2011) is considering that the battery state of energy at the beginning and the end of the trip is roughly the same (note that this assumption can be extremely unrealistic when short displacements are considered; some authors (Sciarretta et al., 2004; Sciarreta and Guzzella, 2007) propose as an alternative to add a penalty for compensating the deviations in the battery charge at the end of the trip). Consequently, the only contributing term in (1) is the fuel power $P_{f}$. Therefore, minimising fuel consumption is equivalent to minimise energy consumption, fuel cost or $\mathrm{CO}_{2}$ emissions (as far as a net quantity of energy is required). Although the usual option in the HEV control bibliography is considering $\alpha=0$, any arbitrary selection of $\alpha$ will lead to the same solution if charge sustainability is imposed (i.e. the integral of the battery power $P_{b}$ over the cycle is zero). 
If the depletion of the battery is allowed, as in the case of PHEVs, the selection of the optimisation objective is of major importance. Since two energy sources are available (electricity from the grid and fuel), a weighted sum of the two concepts may be considered in order to define a metric proportional to the overall energy consumption, cost or emissions. Four different options, summarised in Table 1, are next discussed.

If the goal is to minimise the vehicle energy consumption, both fuel and battery power should be considered and they can be directly summed $(\alpha=1)$. The main drawback of this approach is that primary energy consumption can importantly differ from the direct addition, since electricity and fuel have different production and distribution efficiencies.

Hence, in order to consider the primary energy consumption, $\alpha$ should represent the ratio between the primary energy cost of the engine fuel and the energy stored in the battery $\alpha=\epsilon_{b} / \epsilon_{f}$, where $\epsilon$ corresponds to the inverse of the well-to-tank efficiency for the battery stored energy (referred with subscript $b$ ), and the fuel $(f)$. In case both grid electricity and engine fuel are produced from the same source (e.g. petroleum), using $\alpha=\epsilon_{b} / \epsilon_{f}$ would lead to the overall energetic optimal; however, in many cases different energy sources are used in the electricity generation, and the primary energy cost of the fuel and electricity may not be comparable. In this case, availability or strategic factors may be of major importance.

Another possibility for the cost index is the $\mathrm{CO}_{2}$ footprint. Accordingly, $\alpha$ must adopt the value $\mu_{b} / \mu_{f}$, where $\mu$ refers to the $\mathrm{CO}_{2}$ emissions attached to the electricity production or the fuel burning (e.g. in $\mathrm{g}$ of $\mathrm{CO}_{2} \mathrm{per} \mathrm{kWh}$ ). This selection of $\alpha$ explicitly considers the $\mathrm{CO}_{2}$ emissions during the vehicle operation. In this line, some authors have also proposed including in the cost index (1) the emission of other pollutants, as $\mathrm{NO}_{x}$ or particulate matter (Nuesch et al., 2012).

Finally, overall economic operation cost may be minimised, which results of high interest for the end user. For that, $\alpha$ must be equal to the ratio between $\chi_{b} / \chi_{f}$, where $\chi$ is the energy price of electricity or fuel.

Note that the values of the energy price $\chi, \mathrm{CO}_{2}$ emission $\mu$, and primary energy consumption $\epsilon$, strongly depend on the regional energy market and the share of different technologies in the electrical production, and the selected value will affect the optimal solution.

Table 1. Value of $\alpha$ according to different cost metrics

\begin{tabular}{cl}
\hline$\alpha=0$ & HEV \\
$\alpha=1$ & PHEV vehicle energy \\
$\alpha=\epsilon_{b} / \epsilon_{f}$ & PHEV well-to-wheel energy \\
$\alpha=\chi_{b} / \chi_{f}$ & PHEV economic cost \\
$\alpha=\mu_{b} / \mu_{f}$ & PHEV $\mathrm{CO}_{2}$ emissions \\
\hline
\end{tabular}

In addition to the cost function, the minimisation problem also includes the following constraints:

$$
\begin{gathered}
E_{b, \text { min }} \leq E_{b}(t) \leq E_{b, \text { max }} \\
P_{m, i, \text { min }}(t) \leq P_{m, i}(u(t), t) \leq P_{m, i, \max }(t) \forall i=1, \ldots, N \\
0 \leq P_{e}(u(t), t) \leq P_{e, \max }(t) \\
P_{b, \text { min }}\left(E_{b}(t), t\right) \leq P_{b}(t) \leq P_{b, \max }\left(E_{b}(t), t\right)
\end{gathered}
$$

where subscript $i$ refers to each of the $N$ electrical machines $m$ (motors and generators) in the powertrain, while the subscript $e$ refers to the internal combustion engine. Other constraints associated to the operation of the different subsystems could be also considered, as emissions during transients or noise and vibration of the engine (Millo et al., 2013). 
Additionally, the following global constraint associated to the charge sustainability should be considered for HEVs:

$$
\int_{t_{0}}^{t_{f}} P_{b}\left(u(t), E_{b}(t), t\right) \mathrm{dt}=0
$$

Finally, the vehicle power requirements $P_{r e q}(t)$ should be attended. The mathematical expression of this last constraint depends on the powertrain architecture, which may exhibit different number of electrical machines and transmission elements. Despite the wide range of existing powertrain configurations (Chan et al., 2009), the series architecture has been chosen in this paper for illustrative purposes because of its simplicity, and because it is being studied for PHEV applications (Emadi et al., 2005). Nevertheless, this arbitrary selection does not reduce the formulation applicability, and the methods presented are independent of the selected configuration; in Guardiola et al. (2012) the same methods have been applied to a parallel configuration.

$$
\text { Series hybrid architecture }
$$

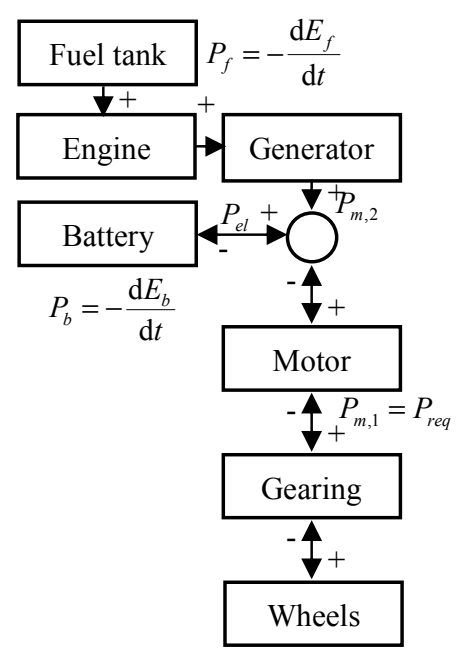

Figure 1. HEV/PHEV powertrain layout, nomenclature and sign criteria.

Figure 1 shows a scheme of the power flow in the series hybrid architecture with the sign criterion used; there the motor is supplied with electrical energy coming from an electric storage system (usually batteries) or alternatively from a generator consisting of an internal combustion engine and an electrical machine (i.e. range extender). In this sense, the engine is not directly coupled to the wheels, thus allowing the engine to operate in a narrow region around its sweet spot or maximum efficiency line. Since $P_{m, 2}+P_{b}$ must provide the electrical input for $P_{m, 1}$, control action may be defined as the electrical output of the generator:

$$
u(t)=P_{m, 2}(t)
$$

Since the vehicle wheels are exclusively driven by an electric motor (or a set of electric motors), the constraint due to the power requirements becomes:

$$
P_{r e q}(t)=P_{m, 1}(t)
$$

\subsection{Implementation details}

For the present work a backward quasi-static approach was employed to model the vehicle dynamics (Rizzoni et al., 1999), which allows the computation of the benchmarking solution based on DP. Engine and motor efficiencies were mapped with rotational speed and torque, while maximum and minimum torques were defined as functions of the speed. Main characteristics for the powertrain architectures used in the HEV and PHEV simulations are summarised 
in Table 2. Basically, the same configuration was used for the PHEV and HEV alike, except for the bigger battery size of the PHEV configuration.

A zero order model was considered for the battery, whose open circuit voltage and equivalent resistance was mapped as function of $S O E$ as shown in Figure 2. In order to analyse the influence of the battery characteristics on the final solution, two different battery chemistries, hereinafter called A and B, were considered. Battery A is based on a NiCd battery, while battery B is based on lithium-ion technology. For both batteries the model parameters were obtained from actual measurements.

Table 2. Description of the main vehicle features

\begin{tabular}{ccc} 
& HEV & PHEV \\
\hline Vehicle mass & $1600 \mathrm{~kg}$ & $1600 \mathrm{~kg}$ \\
Engine power & $75 \mathrm{~kW}$ & $75 \mathrm{~kW}$ \\
Generator power & $60 \mathrm{~kW}$ & $60 \mathrm{~kW}$ \\
Motor power & $160 \mathrm{~kW}$ & $160 \mathrm{~kW}$ \\
Battery power & $80 \mathrm{~kW}$ & $80 \mathrm{~kW}$ \\
Battery energy capacity (A/B) & $6.33 \mathrm{MJ} / 7.82 \mathrm{MJ}$ & $11.02 / 13.61 \mathrm{MJ}$ \\
\hline
\end{tabular}

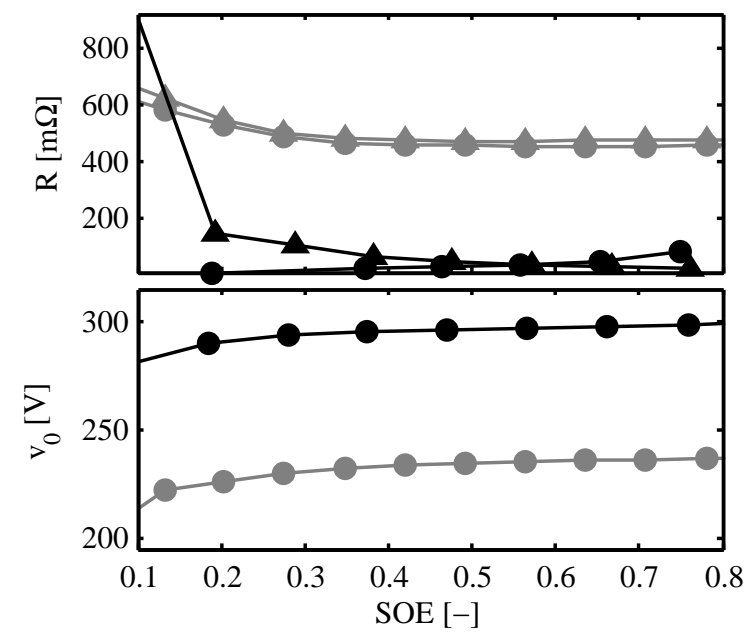

Figure 2. Open circuit voltage and equivalent electrical resistances during charge (circles) and discharge (triangles) and open circuit voltage for battery A (grey) and B (black).

For illustrating different aspects of the research, the NEDC, US06 and FTP75 cycles were considered, whose speed profiles are shown in Figure 3. Because the range of the vehicle is usual higher than the cycles selected, in some simulations several consecutive repetitions of a given cycle are used (the notation 3.US06 is used for 3 repetitions of the US06 cycle).

\section{Energy management strategies}

There is a wide literature on HEV/PHEV control that ranges from rule-based control (Baumann et al., 2000; Schouten et al., 2003; Poursamad and Montazeri, 2008) to the application of the optimal control theory (a few examples may be found in Wei et al. (2007), Bernard et al. (2010), Kermani et al. (2012), and van Keulen et al. (2012)); in some cases, simple implementable rule-based control may be explicitly obtained from the application of the optimal control theory (Lin et al., 2003; Ambühl et al., 2010; Bianchi et al., 2011). 


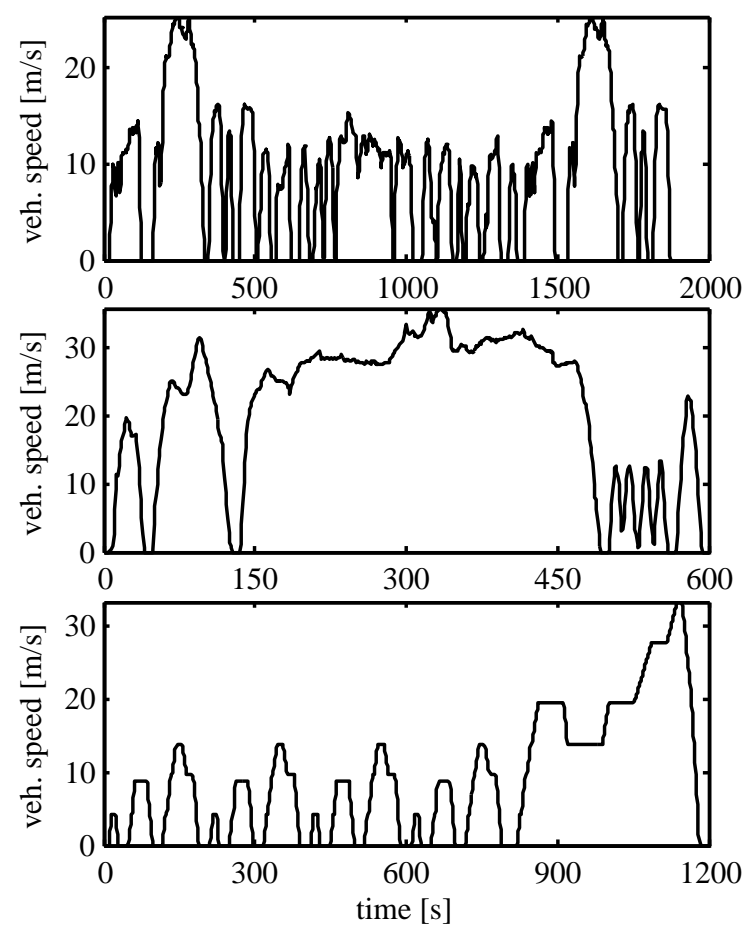

Figure 3. Speed profile of three homologation cycles used for the simulations: FTP75 (top), US06 (centre) and NEDC (bottom).

For the optimal control approach, the a priori knowledge of the driving cycle is usually assumed (although stochastic optimisation concepts have also been applied, as for example in Moura et al. (2011); Chan-Chiao et al. (2004)). Next three usual approaches are described.

\subsection{Dynamic Programming}

The DP algorithm is based on Bellman's principle of optimality, which reads: "An optimal policy has the property that whatever the initial state and initial decision are, the remaining decisions must constitute an optimal policy with regard to the state resulting from the first decision" (Lewis and Syrmos, 1995). The method consists in applying a proper discretisation of the problem in both controls and states; starting from the final state, the DP algorithm determines the optimal control law choosing at each step the control which minimises the cost function subject to the set of constraints. The DP code used in this work is that presented in Sundström and Guzzella (2009); Sundström et al. (2010).

Since the DP uses a non-causal backward approach to solve the optimisation problem, it is assumed that the complete driving profile is known a priori, which prevents its utilisation for on-line control purposes. Nevertheless, the result can be used as a benchmark for the performance of other strategies, or even to derive the tuning parameters required by other methods.

\subsection{Pontryagin Minimum Principle}

The application of the PMP allows to reduce the global optimisation into a local minimisation problem (Delprat et al., 2001; Serrao et al., 2011). The PMP applied to minimising (1) subject to (5)-(8) states that if $u^{*}$ is an optimal solution of the control problem as defined in Section 2 , and $E_{b}^{*}$ is the optimal trajectory of the battery energy over the entire driving profile, then:

$$
H\left(u^{*}, E_{b}^{*}, \lambda^{*}, t\right) \leq H\left(u, E_{b}^{*}, \lambda^{*}, t\right) \forall u \in U, t \in\left[t_{0}, t_{f}\right]
$$


where $U$ is the space of possible control actions, and $H$ is the Hamiltonian function for the optimal problem (1), subject to (4)-(8), defined as:

$$
H=P_{f}+\alpha P_{b}-\lambda \dot{E}_{b}=P_{f}+(\alpha+\lambda) P_{b}
$$

Note that the PMP provides necessary conditions of optimality of a given control trajectory. Moreover, if the optimisation problem admits a unique solution, that provided by the PMP is the optimal solution (Kim et al., 2011), leading to use the necessary conditions of optimality as sufficient conditions to seek for the optimum. It is also interesting to note that in equation (12), and as a direct consequence of selecting $E_{b}$ as the state variable, $P_{f}$ and $P_{b}$ share the same units, and hence $\alpha$ and the Lagrange multiplier $\lambda$ are dimensionless. On the other hand, while $\alpha$ is a constant related to the parameter to optimise, the PMP provides conditions to define the evolution of $\lambda$ :

$$
\dot{\lambda}=\frac{\partial H}{\partial E_{b}}=(\alpha+\lambda) \frac{\partial P_{b}}{\partial E_{b}}=(\alpha+\lambda) P_{e l} \frac{\partial\left(P_{b} / P_{e l}\right)}{\partial E_{b}}
$$

where $P_{e l}$ is the electrical power provided by the battery. Note that, according to Figure $1, P_{e l}$ only depends on the required power and the power split between the generator and the battery (which is imposed through $u(t)$ ):

$$
P_{e l}=P_{r e q}-P_{m, 2}=P_{r e q}-u
$$

and consequently depends on $u(t)$ and $t$, but not on $E_{b}$. However, as the battery behaviour is affected by its state of energy, this statement is not true for $P_{b}$; the ratio $\eta_{b}=P_{b} / P_{e l}$ corresponds to the battery efficiency (or its inverse) when charging (or discharging) and depends on the battery energy level due to the variation of the battery characteristics as shown in Figure 2. Consequently, (13) may be rewritten as:

$$
\dot{\lambda}=(\alpha+\lambda) P_{e l} \frac{\partial \eta_{b}}{\partial E_{b}}
$$

This last expression is implementable if the battery efficiency is mapped with $E_{b}$ and $P_{e l}$. Thus, given a time discretisation of the problem and an initial value for the Lagrangian multiplier $\lambda_{0}=\lambda\left(t_{0}\right)$, the evolution of $\lambda$ may be computed:

$$
\lambda_{i}=\lambda_{i-1}+\left(\alpha+\lambda_{i-1}\right) P_{e l, i-1} \frac{\partial \eta_{b}\left(P_{e l, i-1}, E_{b, i-1}\right)}{\partial E_{b}}\left(t_{i}-t_{i-1}\right)
$$

and the optimal control policy calculated through the minimisation of (12) at each time step.

Despite (16) superficially seems to be independent of the driving cycle, the optimal value of $\lambda_{0}$ depends on the considered cycle and constraints. This issue can be partially solved through the application of adaptive methods (Chasse et al., 2010).

Additionally, if the final state is not restricted, $\lambda\left(t_{f}\right)=0$ (Pontryagin et al., 1962; Naidu, 2003), while if the terminal value of the state is constrained as in

$$
E_{b, \min } \leq E_{b}\left(t_{f}\right) \leq E_{b, \max }
$$

and such restriction becomes active, then $\lambda\left(t_{f}\right) \neq 0$. PMP also allows considering constraints in the state variable evolution, as (4) is, through the use of penalty functions (Stockar et al., 2011), interior point constraints or slack variables (Naidu, 2003).

\subsection{Equivalent Consumption Minimization Strategy}

The ECMS was introduced in Paganelli et al. (2001) as a method to solve the energy management problem in charge sustaining HEV. The idea underlying ECMS is that, as grid energy is not used for recharging the battery, all the energy used comes ultimately from fuel, so it is possible to define a cost for the battery usage in order to take into account the potential of discharging the batteries in the current moment and recharge them in the future or vice versa. In this way, it is possible to define an instantaneous cost which takes into account both fuel and electric energy consumption, for instance:

$$
f=P_{f}+s P_{b}
$$


where the parameter $s$ is traditionally defined as an equivalent factor between fuel and battery energy sources. From a mathematical point of view, ECMS can be seen as a simplification of the PMP approach in which the Lagrangian multiplier $\lambda$ is constant over the driving cycle (Serrao et al., 2009); according to (13) this will be exactly true if the battery efficiency is independent of the battery energy level, thus is a reasonable simplification in most cases.

As in the case of PMP, the optimal value of the parameter $s$ depends on the driving profile; nevertheless, different adaptive methods have been developed for mitigating this issue (Musardo et al., 2005; Onori et al., 2010).

\section{Control strategy tuning through the $\lambda$-plot method}

Although both ECMS and PMP may be implemented in a forward looking simulator, their optimality is not guaranteed online, since their behaviour is strongly dependent on the selection of the initial value of $\lambda\left(t_{0}\right)$, and $s$ respectively. Additionally, since these values depend on the driving cycle, their optimal value cannot be obtained a priori. The issue of obtaining the initial value of $\lambda$ can be solved by means of a shooting method (Serrao et al., 2011); however, if the optimal solution is available (through DP or otherwise), this information can be used for inferring the initial value by means of the $\lambda$-plot method presented in Guardiola et al. (2012), which was developed exclusively for $\alpha=0$, but that can be slightly modified for coping with the more general problem. The underlying idea is that, according to (11), the optimal Lagrange multiplier $\left(\lambda^{*}\right)$ must satisfy along the whole optimal trajectory $\left(\partial H / \partial P_{b}\right)_{o p t}=0$, what applied to $(12)$ yields to:

$$
\lambda^{*}(t)=-\left(\frac{\partial P_{f}}{\partial P_{b}}\right)_{o p t}-\alpha
$$

However, due to the existence of singular points and saturations, $P_{b}$ or $P_{f}$ may not be differentiable in some points of their domains, and also $\left(\partial P_{f} / \partial P_{b}\right)$ may depend on the sign of the considered variation $\delta P_{b}$. To deal with this issue, numerical differentiation of the optimal solution is done by considering variations of different size in the control action $\delta u$, and analysing their effect on the battery power $\delta P_{b}$, and on the fuel power $\delta P_{f}$; in all cases the satisfaction of the different constraints must also be checked. This allows to compute $\lambda^{+}(t)=\max \left(-\delta P_{f} / \delta P_{b}\right)$ with $\delta P_{b}>0$, and $\lambda^{-}(t)=\min \left(-\delta P_{f} / \delta P_{b}\right)$ with $\delta P_{b}<0$, which provide a manifold where the value of the $\lambda^{*}$ trajectory should be contained:

$$
\lambda^{+}(t) \leq \lambda^{*}(t)+\alpha \leq \lambda^{-}(t)
$$

Figure 4 shows an example of the results obtained during simulations with battery A and a HEV configuration in both NEDC and US06 cycles; the DP solution has been locally differentiated in order to provide estimates for $\lambda^{+}(t)$ and $\lambda^{-}(t)$, marked in the top plots as + and $\cdot$ respectively. The precision of the $\lambda^{+}$and $\lambda^{-}$estimates depends on the numerical differentiation used, and also on the precision of the original DP solution; in general the resolution in the numerical differentiation $\left(\delta P_{b}\right)$ should be kept in the same order of magnitude of the discretization used in the DP solution. On the other hand, if a saturation is reached with $\delta P_{b}>0, \lambda^{+}$may not be calculated because there are no feasible points and $\lambda^{-}$provides an abnormal high value; this is due to the fact that the constraint in the saturated variable is restricting the solution. Correspondingly, $\lambda^{+}$exhibits low values when the solution is not feasible with $\delta P_{b}<0$.

Initial values of $\lambda^{+}(t)$ and $\lambda^{-}(t)$ provide an interval for $\lambda\left(t_{0}\right)$ (as for the HEV case $\alpha=0$ ), which may be used as an initial guess for $\lambda\left(t_{0}\right)$ to be used for PMP simulation (some fine tuning through shooting may be still necessary, but the search interval is bounded). Figure 4 also shows the trajectory of $\lambda$ for the PMP simulation if such initial value is used. Despite the existence of few outliers, $\lambda$ trajectory is always within the $\left[\lambda^{+}, \lambda^{-}\right]$range, and the evolution of the battery $S O E$ for the PMP strategy is almost identical to that of the DP solution, as depicted in the lower plots of Figure 4. This illustrates that the PMP tuned through the so called $\lambda$-plot method replicates the DP solution, which is assumed to be optimal. The few detected outliers are probably related with (limited) non-convexities in the problem, which can be consequence of the local restrictions, saturations or the time discretisation considered; however, this issue clearly exceeds the scope of the present paper (note that the optimisations methods used are based on the convexity assumption). 


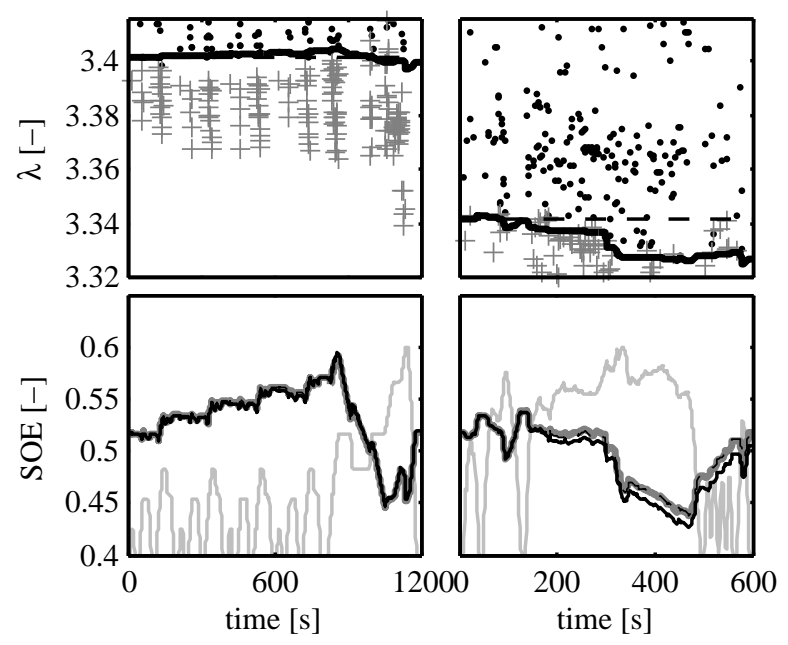

Figure 4. Evolution of the $\lambda$ parameter during the NEDC (left) and US06 cycle (right) inside the manifold provided by $\lambda^{-}(\cdot)$ and $\lambda^{+}(+)$for a HEV $(\alpha=0)$; dashed line represents the value of $s$ used for the ECMS. In lower plots, the evolution of the $S O E$ calculated with DP (dashed black), PMP (grey) and ECMS (black) is presented; driving profile is also shown for reference (light grey).

As shown in the top plots of Figure 4, $\lambda$ variation during the cycle is not significant. Hence using a constant parameter $s$ (dashed line in top plots) with the ECMS approach would provide similar results. The manifold $\left[\lambda^{+}, \lambda^{-}\right]$ also provides a good initial estimate of $s$. Results of the $S O E$ trajectory for the ECMS are shown in bottom plots of Figure 4; as one could expect ECMS provides a solution almost identical to the one of the DP and the PMP in the NEDC cycle (left plots), since there the variation of $\lambda$ is lower. For the US06 cycle (right plots) although the $S O E$ trajectory is quite similar, some differences can be appreciated.

\section{Results and discussion}

\subsection{Differences between HEV and PHEV control and $\alpha$ effect}

For investigating the effects of the differences between the HEV and PHEV control, several simulations were run for the three repetitions of the studied cycles with the PHEV configuration and varying the value of $\alpha$ (for HEV $\alpha=0$ was considered and the charge sustainability was imposed). DP solution was computed in all cases, and both PMP and ECMS were tuned on the basis of the $\lambda$-plot derived from the DP using the approach sketched in Section 4.

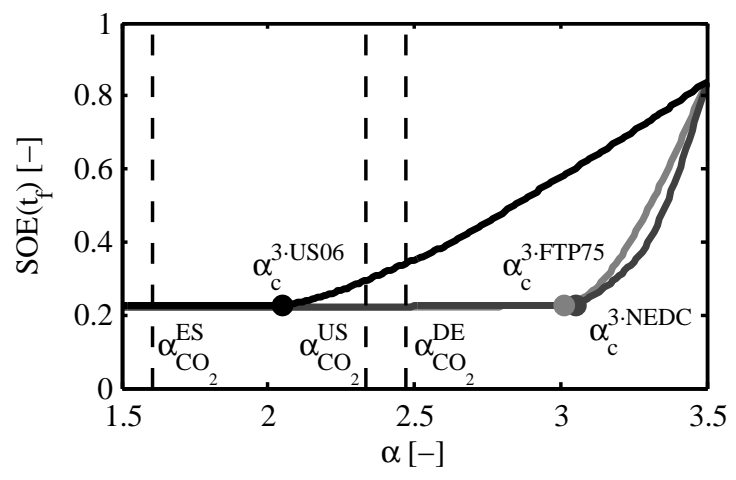

Figure 5. Effect of the $\alpha$ parameter on the SOE at the end of the 3.US06 (black), 3.NEDC (dark grey) and 3.FTP75 (light grey); critical values $\alpha_{c}$ are marked with a coloured circle. Dashed lines show the $\alpha$ values according to $\mathrm{CO}_{2}$ minimisation in Spain (ES), United States of America (US) and Germany (DE). 
Figure 5 shows the results of the final battery state of energy for the three cycles when varying the value of $\alpha$. Results are shown for the PMP simulation once $\lambda\left(t_{0}\right)$ was adjusted; DP results are not shown but are almost identical. As it can be clearly noticed, for low values of $\alpha$, the solution is limited by the battery minimum charge; this situation corresponds to depleting the battery, and since in that situation the amount of net energy coming from the battery is kept constant, the solution is not sensitive to variations of $\alpha$. For all these cases $\operatorname{SOE}\left(t_{f}\right)=\operatorname{SOE} E_{\text {min }}$. For values of $\alpha$ higher than a critical value $\left(\alpha_{c}\right)$, the restriction in the end state $\left(E_{b}\left(t_{f}\right)\right.$ or its equivalent $\left.S O E\left(t_{f}\right)\right)$ is no longer active, and then the solution is sensitive to the selected value of $\alpha$.

Figure 6 shows two examples of the evolution for the 3.NDEC cycle, with a value of $\alpha$ lower (left) and higher (right) than $\alpha_{c}$. It can be appreciated that with $\alpha>\alpha_{c}$ (right plot) the final state of the battery is higher than the minimum allowed $\left(S O E_{\min }=0.225\right)$, and then the problem solution corresponds to the optimum of the unconstrained problem (i.e. the problem without considering the terminal state constraint (17)). Accordingly, the terminal value of the Lagrange multiplier $\lambda\left(t_{f}\right)$ is zero $\left(\lambda\left(t_{f}\right)+\alpha=\alpha=3.25\right.$, as shown in the right $\lambda$-plot of Figure 6).

On the other hand, with $\alpha<\alpha_{c}$ as in the left-hand plots on Figure $6, \lambda\left(t_{f}\right)$ gets a positive value $\left(\lambda\left(t_{f}\right)+\alpha>\alpha\right)$, that is needed for forcing the state to fulfil the restriction of the final state (17). In that case, the solution of the problem is qualitatively similar to that of the HEV energy management, considering the following restriction instead of (8):

$$
E_{b}\left(t_{0}\right)-E_{b}\left(t_{f}\right)=\int_{t_{0}}^{t_{f}} P_{b}\left(u(t), E_{b}(t), t\right) \mathrm{dt}=E_{b}\left(t_{0}\right)-E_{b, \min }
$$

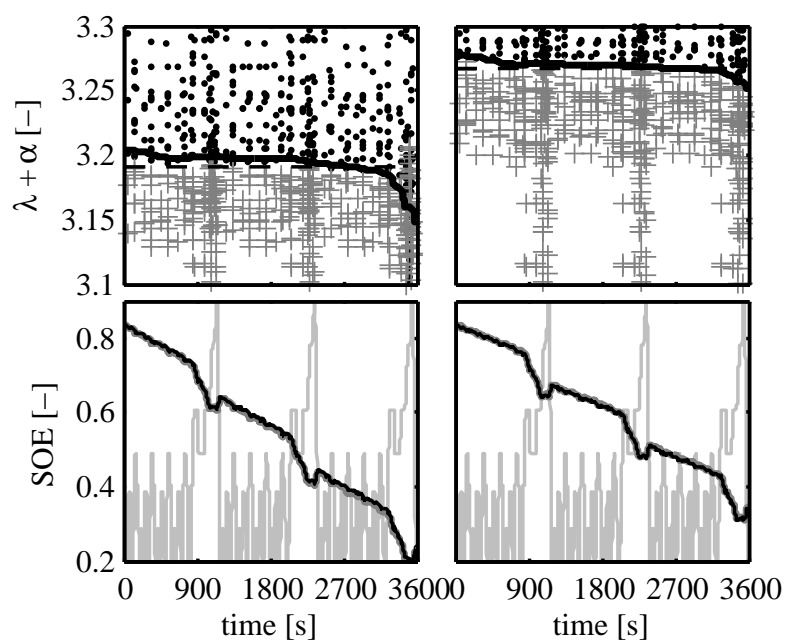

Figure 6. Evolution of $\lambda$ and $S O E$ in the 3.NDEC cycle for the PHEV considering $\alpha=3$ (left) and $\alpha=3.25$ (right).

For investigating the transition between the constrained and the unconstrained solution of the original problem, the optimisation problem was solved without considering the restriction on the minimum energy in the battery. This was done by DP for several values of $\alpha$, and then PMP was tuned for replicating the DP solution in order to provide insight on $\lambda\left(t_{f}\right)$. Figure 7 depicts the values obtained when (17) was not considered (circles) and the solution of the constrained problem (squares). As in the case of Figure 5, here the solution of the problem with the constraint on the final state also exhibits a transition between the solution of the unconstrained problem (for $\alpha>\alpha_{c}$, marked in the figure with a black square), and the solution of the problem satisfying (21).

It is clear the transition between the two problems, and that the value of $\lambda\left(t_{f}\right)$ is a metric of the effort needed for fulfilling the final state constraint. This fact is coherent with the PMP theory (Naidu, 2003), since if no restriction is considered in the final state $\lambda\left(t_{f}\right)=0$, while when the restriction is active, $\lambda\left(t_{f}\right)$ gets the value that ensures $H\left(t_{f}\right)=$ 0 . As expected, in the case of the solution to the unconstrained problem, marked with circles in the Figure, $\lambda\left(t_{f}\right)$ approaches 0 (actual values are slightly different to 0 , but it is attributed to the numerical precision and discretization errors). 
The critical value $\alpha_{c}$ corresponds to the transition between the two situations, and consequently the solution for this case must satisfy both $E_{b}\left(t_{f}\right)=E_{b, \min }$ and $\lambda\left(t_{f}\right)=0$.
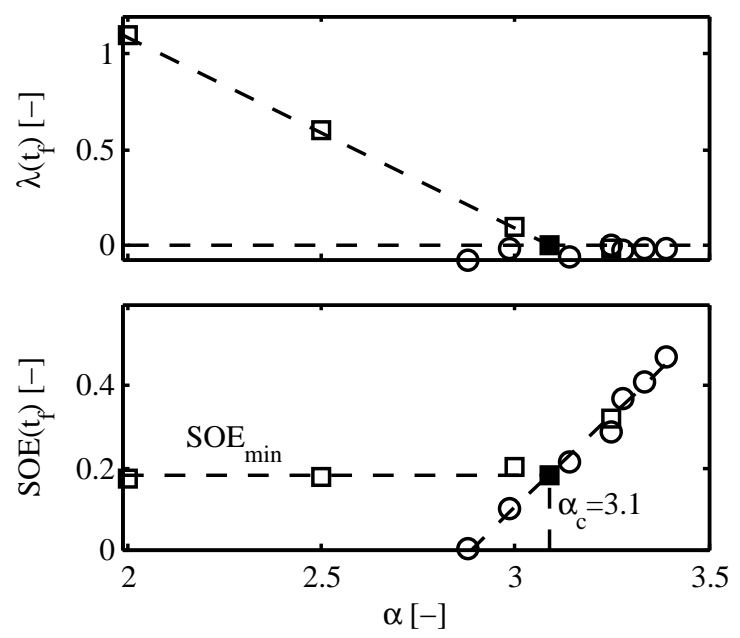

Figure 7. Value of $\lambda\left(t_{f}\right)$ (top) and $S O E\left(t_{f}\right)$ bottom obtained with PMP for several values of $\alpha$ considering (squares) or not (circles) the battery energy constraint in the $3 \cdot$ NEDC cycle.

\subsection{Comparing fuel, energy and $\mathrm{CO}_{2}$ minimisation}

On the basis of the precedent discussion, it is clear that there is a shift in the system behaviour according to the considered value of $\alpha$ with regards to the critical $\alpha_{c}$. As stated above, for $\alpha<\alpha_{c}$, the final state of the battery is an active constraint, and hence the solution is the same regardless of the cost to be minimised. This is similar to the situation that occurs with the HEV, where the total amount of energy provided by the battery is constant $(0$, in the case of $\mathrm{HEV} ; E_{b}\left(t_{0}\right)-E_{b, \min }$ for the PHEV vehicle). In this case there is no difference at all regardless of the cost to be optimised.

However, if $\alpha>\alpha_{c}$ (i.e. the battery is not depleted during the operation), the selection of the cost function clearly impacts the solution. In this case, optimising fuel consumption, energy or $\mathrm{CO}_{2}$ is no longer equivalent. The effect of the selection of $\alpha$ has been shown in Figure 5.

According to Table 1, $\alpha$ depends not only on the cost to be minimised, but also on regional parameters including energy generation and distribution costs (and taxing) and carbon intensity in the energy production (energy generation mix). Figure 8 shows the value of $\alpha$ according to two different criteria (economic cost and $\mathrm{CO}_{2}$ emission) for different countries; it may be noticed that there is a huge variation among countries. In countries like France or Sweden, where the share of the nuclear or renewable energy on the electricity generation mix is very high, using $\alpha_{\mathrm{CO}_{2}}$ implies using an almost pure electrical solution (combustion engine is only used after the battery depletion); because electricity cost is also comparatively low, $\alpha_{\text {cost }}$ is also quite low, and the resulting solution is similar. However, in most countries $\alpha_{\text {cost }}<\alpha_{\mathrm{CO}_{2}}$ (dashed line in Figure 8 shows the equivalence between both factors), and optimal economic cost (presumably end user objective) will increase the electrical energy consumption beyond the $\mathrm{CO}_{2}$ optimal solution. This issue could be corrected through taxation or regulation if the final goal is the global warming control.

As an example, $\alpha_{\mathrm{CO}_{2}}$ for three different countries has been marked in Figure 5. Note that the optimal solution with regards to $\mathrm{CO}_{2}$ minimisation in the US06 cycle is shifted depending of the considered country, although in the rest of the cycles $\alpha_{\mathrm{CO}_{2}}<\alpha_{c}$ and all the solutions will be equal regardless of the considered country. Because in all countries $\alpha_{\text {cost }}<\alpha_{c}$, the optimal solution considering economic cost will be that depleting the battery.

\subsection{Effect of the battery size on the solution and optimal battery sizing}

It is important to note that the value of $\alpha_{c}$ depends on the driving cycle characteristics and the battery size: driving profiles with lower requirements or bigger batteries cause $\alpha_{c}$ to diminish. This is because in that situations the battery 


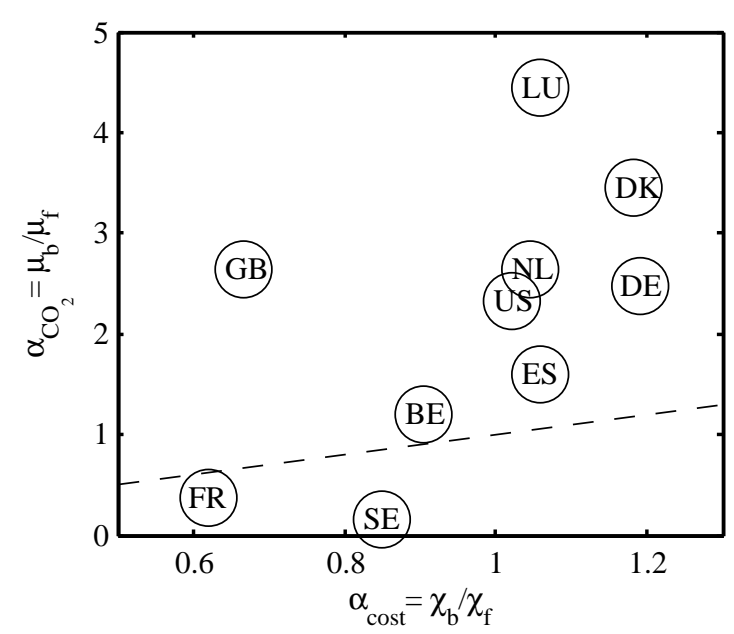

Figure 8. Values of $\alpha$ considering cost $\left(\chi_{b} / \chi_{f}\right)$ or $\mathrm{CO}_{2}$ emission $\left(\mu_{b} / \mu_{f}\right)$ for different countries. Data from EABEV (2008).

energy limitations are less stringent and the system is allowed to operate in the global optimum (rather than the optimum of the problem constrained by the terminal condition); this is the case of the 3.US06 cycle (with lower energy demand, despite higher power requirements) when compared to the $3 \cdot$ FTP75 or 3·NEDC cycles, as shown in Figure 5.

In order to evaluate the effect of the battery size on the solution, 3-NEDC and 3-FTP cycles were run with different values of $\alpha$ and batteries of different capacities, ranging from the original battery of the considered PHEV configuration (12 Ah) to an infinite capacity battery. Figure 9 summarises the results of such simulations. If the infinite battery is considered, a characteristic line for the cycle may be obtained, which provides the battery energy consumed for a given $\alpha$. As expected, this line depends on the considered cycle, and for very low $\alpha$ values gets saturated ( $\alpha=2.1$ for the 3.FTP and $\alpha=1.3$ for the 3.NEDC): it corresponds to a pure electrical solution and the battery provides all the needed energy for running the cycle.
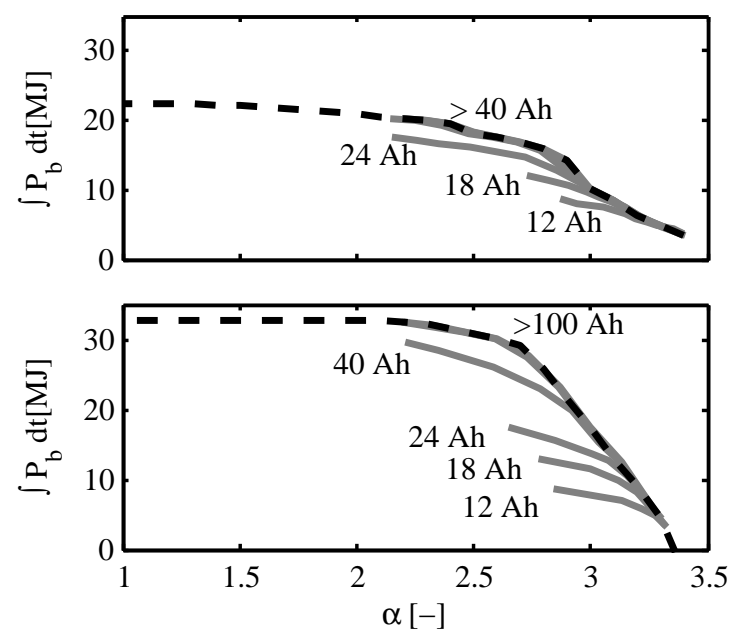

Figure 9. Consumed battery energy during 3·NEDC (top) and 3·FTP (bottom) with different values of $\alpha$ and battery capacities. Black dashed line corresponds to an infinite capacity battery.

In the case of finite size batteries, they behave as the infinite size battery if high values of $\alpha$ are considered. 
However, when the battery efficiency drops as it is being depleted, its characteristic line goes below that of the infinite battery. $\alpha_{c}$ depends on the battery, and it is defined on the basis of the minimum allowable $S O E$ and the battery characteristic line.

Correspondingly, given a value of $\alpha$ and a driving cycle, it is possible to derive the minimum size of the battery that allows operating in the global optimum. Despite many other criteria are to be evaluated, this could be of interest as a design criterion for sizing the battery for a given application.

Figure figBatSize provides a good framework for the battery selection. For example, for the case of the 3·FTP cycle and $\alpha=3$, any battery with a capacity lower than 18 Ah would be depleted (i.e. $\alpha<\alpha_{c}$ ), while a battery of $18 \mathrm{Ah}$ will be able to operate without activating the terminal restriction. However, its efficiency will be lower than that of a higher capacity battery. Going to 40 Ah capacity would allow operating in a situation near of the ideal infinite capacity battery.

Note that the initial parts of the characteristic lines for all battery sizes collapse in the infinite capacity battery line. This provides the possibility of extrapolating the results between batteries of different sizes. Figure 10 represents the divergence between the behaviour of the infinite capacity battery and the different batteries simulated. It may be seen that approaching (or extrapolating) the results between the different batteries is only possible when $S O E>0.3$, which corresponds to the battery linear zone.
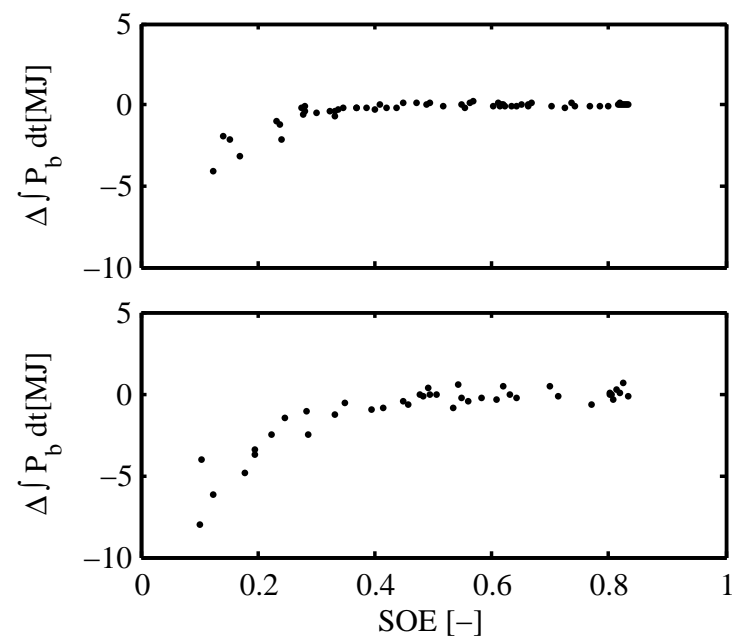

Figure 10. Divergence with regards to an infinite capacity battery as a function of the final SOE for $3 \cdot$ NEDC (top) and $3 \cdot$ FTP (bottom).

\subsection{Effect of the battery chemistry on the solution}

Since battery properties affect the powertrain efficiency, they impact on the vehicle optimal behavior. Figure 11 shows the evolution of the optimal SOE trajectory during the NEDC for the HEV with two different batteries. It can be observed how battery $\mathrm{B}$, with lower equivalent resistance and higher open circuit voltage (so higher efficiency) allows a wider range of variations in the $S O E$ during the driving profile, then taking advantage of the higher efficiency of the electrical powertrain. Regarding the evolution of the $\lambda$, it shows a quite flat behavior due to the low variations in the equivalent resistance and open circuit voltage in the operating range when varying the battery charge level (see Figure 2). However, $\lambda$ values with battery A are higher due to its lower efficiency (the use of the electrical energy path involves a higher cost due to the lower battery efficiency). As it can be noticed in Figure 11, the flatter the behaviour in $\lambda$, the most the ECMS behaviour fits that of the PMP and DP.

5.5. Hints on the PMP and ECMS tuning by shooting for PHEV

PMP and ECMS tuning by shooting has been widely used for the case of HEV, because the terminal value of the battery energy is known. However shooting reference is not clear when the system final state is unknown, as may occur in the PHEV control problem (Stockar et al., 2011). As it has been demonstrated, the solution for PHEV is quite similar in the cases where the final battery energy constraint is activated (the battery is depleted), and then 


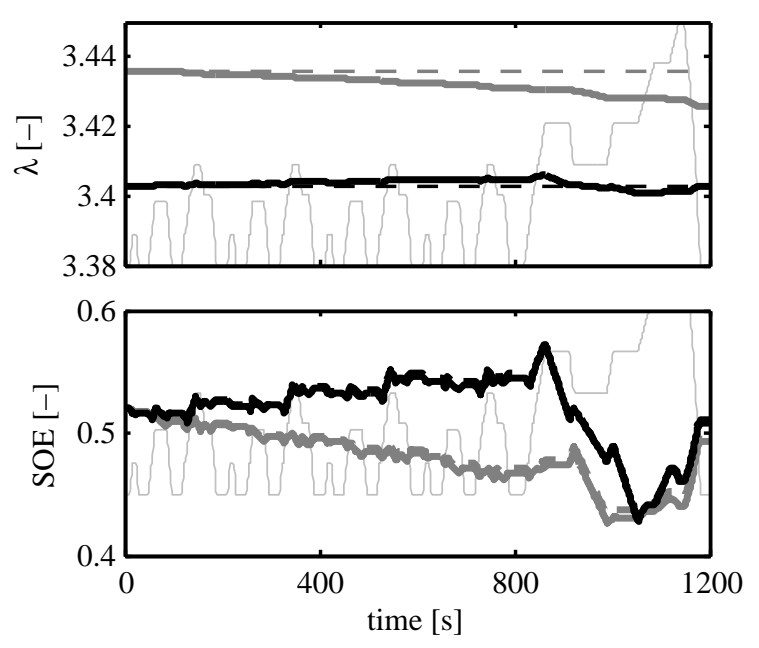

Figure 11. Evolution of the SOE (lower plot) and optimal $\lambda$ (upper plot) for the NEDC cycle obtained with PMP (continuous line) and ECMS (dotted line) approaches during tests with battery A (grey) and B (black).

usual shooting methods considering $E_{b}\left(t_{f}\right)=E_{b, \min }$ can be used. However, in the cases where the optimal solution corresponds to $E_{b}\left(t_{f}\right)>E_{b, \min }$ no shooting reference is available. According to the discussion in the present paper, PMP can be then tuned using $\lambda\left(t_{f}\right)=0$ as shooting objective.

A two-step algorithm can be done then for tuning the PMP (and ECMS likewise): initially solving the unconstrained problem by shooting for $E_{b}\left(t_{f}\right)=E_{b, \min }$; then, if the resulting $\lambda\left(t_{f}\right)$ is negative, shooting for $\lambda\left(t_{f}\right)=0($ the reverse procedure can be also used: searching $\lambda\left(t_{f}\right)=0$ and if $E_{b}\left(t_{f}\right)<E_{b, \min }$, then shooting for $\left.E_{b}\left(t_{f}\right)=E_{b, \min }\right)$.

For the ECMS calibration the problem is similar: if the constraint in the battery size is active then it can be solved like in HEV by shooting for $E_{b}\left(t_{f}\right)=E_{b, \min }$. On the other hand, in the case the $\alpha$ value yields to a global optimum, then the final state is unknown; however, because terminal value of $\lambda\left(t_{f}\right)=0$, then $s=\alpha$ will be a good approximation of the optimum (note that if the variation of the battery efficiency with the battery energy level is high, ECMS is no longer able to provide the optimal solution).

\section{Conclusions}

A formulation of the Energy Management Problem for both HEV and PHEV able to deal with different cost indexes has been presented, and the $\lambda$-plot method has been extended for fitting the formulation. The $\lambda$-plot method allows to obtain a suitable tuning of the ECMS and PMP control strategies from the differentiation of the optimal solution provided by the DP. Despite sometimes a fine tuning of $s$ and $\lambda_{0}$ parameters is required, the $\lambda$-plot method provides a narrow range to search within. In any case, the method advantages go beyond the co-state estimation, since the $\lambda$-plots provide insight into the optimal solution, and shows the conditions in which ECMS is equivalent to PMP. Outliers in the plot also reveal non-convexities in the problem which prevent both strategies from reaching DP results.

The study of different cases has contributed to the understanding of the differences between the HEV and PHEV optimization, mainly related to the final state constraint. In the same line, it has been demonstrated that in the case of PHEVs, the minimisation of different cost functions is equivalent when the battery final state of energy is constraining the solution; however, optimal trajectories differ for the cases where constraint on the final battery state of energy is not activated. Accordingly, a method has been presented for estimating the battery size which allows a vehicle to operate in the global optimum for a given cycle and a given ratio between electricity and fuel costs $(\alpha)$. Finally, the effects of the battery parameters have been studied, showing that for a given driving cycle the $S O E$ evolution and therefore the operating range of the engine are strongly affected by the battery efficiency curve. 


\section{Appendix A. Equivalence between alternative formulations}

\section{Appendix A.1. Selection of the state variable}

In the present paper the battery energy $E_{b}$ has been selected as state variable in the problem formulation; however, this is not by far the most used variable. More usual selections are the battery state of energy ( $S O E)$ or state of charge $(S O C)$. The first one is obtained through the normalisation of the battery energy with respect to the maximum stored energy $\left(S O E=E_{b} / E_{b, \max }\right.$ ), while the second considers electrical charge normalised with respect to the maximum capacity, rather than energy.

The advantage of using $E_{b}$ (and its time derivative $-P_{b}$ ) instead of $S O E$ or $S O C$ is based on the dimension coherence in (12), that causes $\alpha$ and $\lambda$ to be dimensionless and easily interpretable. However, the developed method is general and with a few modifications can be directly applied to alternative formulations employing $S O E$ (with $\left.H^{\prime}=P_{f}-\left(\lambda^{\prime}+\alpha^{\prime}\right) S \dot{O} E\right)$ or $S O C\left(H^{\prime \prime}=P_{f}-\left(\lambda^{\prime \prime}+\alpha^{\prime \prime}\right) S \dot{O} C\right)$. Both formulations are quite common in the literature when dealing with HEV control (i.e. $\alpha=0)$ (Guzzella and Sciarretta, 2005).

For the first, it suffices to slightly modify (13):

$$
\dot{\lambda}^{\prime}=\frac{\partial H^{\prime}}{\partial S O E}=\left(\lambda^{\prime}+\alpha^{\prime}\right) \frac{P_{e l}}{E_{b, \max }} \frac{\partial \eta_{b}}{\partial S O E}
$$

For the second formulation, the relationship between the stored energy and the battery charge must be considered. In the case of the zero order model it stands:

$$
\frac{\partial S O C}{\partial S O E}=\frac{v_{00}}{v_{0}}
$$

where $v_{0}$ is the open circuit voltage of the battery for the given energy level, and $v_{00}$ is its value at maximum battery level $(S O C=S O E=1)$. Then, (13) results:

$$
\dot{\lambda}^{\prime \prime}=\frac{\partial H^{\prime \prime}}{\partial S O C}=\left(\lambda^{\prime \prime}+\alpha^{\prime \prime}\right) \frac{P_{e l}}{E_{b, \max }} \frac{v_{00}}{v_{0}} \frac{\partial \eta_{b}}{\partial S O C}
$$

Appendix A.2. Selection of the cost function

Alternatively, (1) can be written as:

$$
J^{\prime \prime \prime}=\alpha\left(E_{b}\left(t_{0}\right)-E_{b}\left(t_{f}\right)\right)+\int_{t_{0}}^{t_{f}} P_{f}\left(u(t), E_{b}(t), t\right) \mathrm{dt}
$$

and its associated Hamiltonian results:

$$
H^{\prime \prime \prime}=P_{f}+\lambda^{\prime \prime \prime} P_{b}
$$

It is straightforward deducing that the graphical method may be applied to this problem (i.e. $\lambda^{+} \leq \lambda^{\prime \prime \prime} \leq \lambda^{-}$). As it can be easily deduced, there is direct relationship between the new Lagrange multiplier and that of the initial formulation in Guardiola et al. (2012):

$$
\lambda^{\prime \prime \prime}=\lambda+\alpha
$$

On the other hand, when the final value of the state is not forced, and according to the PMP, then:

$$
\lambda^{\prime \prime \prime}\left(t_{f}\right)=-\frac{\partial\left(\alpha\left(E_{b}\left(t_{0}\right)-E_{b}\left(t_{f}\right)\right)\right)}{\partial E_{b}\left(t_{f}\right)}=\alpha
$$

which is equivalent to $\lambda\left(t_{f}\right)=0$.

Note that the discussion in section 5.5 is still valid but the critical value of $\lambda^{\prime \prime \prime}\left(t_{f}\right)$ for shifting between the two shooting problems is $\alpha$ (and the shooting objectives become $E_{b}\left(t_{f}\right)=E_{b, \min }$ or $\lambda^{\prime \prime \prime}\left(t_{f}\right)=\alpha$, alternatively). 


\section{References}

D. Ambühl, O. Sundström, A. Sciarretta, and L. Guzzella. Explicit optimal control policy and its practical application for hybrid electric powertrains. Control Engineering Practice, 18(12):1429 - 1439, 2010. ISSN 0967-0661.

T. Banjac, F. Trenc, and T. Katrašnik. Energy conversion efficiency of hybrid electric heavy-duty vehicles operating according to diverse drive cycles. Energy Conversion and Management, 50:2865-2878, 2009.

B. Baumann, G. Washington, B. Glenn, and G. Rizzoni. Mechatronic design and control of hybrid electric vehicles. IEEE/ASME Transactions on Mechatronics, 5(1):58-72, 2000.

J. Bernard, S. Delprat, T. Guerra, and F. Bchi. Fuel efficient power management strategy for fuel cell hybrid powertrains. Control Engineering Practice, 18(4):408-417, 2010. cited By (since 1996)26.

D. Bianchi, L. Rolando, L. Serrao, S. Onori, G. Rizzoni, N. Al-Khayat, T. Hsieh, and P. Kang. Layered control strategies for hybrid electric vehicles based on optimal control. International Journal of Electric and Hybrid Vehicles, 3(2):191-217, 2011.

C. C. Chan, Y. S. Wong, and A. Bouscayrol. Powering sustainable mobility: Roadmaps of electric, hybrid, and fuel cell vehicles. Proceedings of the IEEE, 97(4):603-607, 2009.

L. Chan-Chiao, P. Huei, and J. Grizzle. A stochastic control strategy for hybrid electric vehicles. In Proceedings of the 2004 American Control Conference, Boston, MA, USA, June-July 2004.

A. Chasse, A. Sciarretta, and J. Chauvin. Online optimal control of a parallel hybrid with costate adaptation. In Proceedings of the 6th IFAC Symposium Advances in Automotive Control, 2010.

S. Delprat, T. Guerra, G. Paganelli, J. Lauber, and M. Delhom. Control strategy optimization for an hybrid parallel powertrain. In American Control Conference, 2001. Proceedings of the 2001, volume 2, pages 1315-1320 vol.2, 2001.

EABEV. Energy consumption, $\mathrm{CO}_{2}$ emissions and other considerations related to battery electric vehicles. Technical report, European Association for Battery Electric Vehicles, 2008.

A. Emadi, K. Rajashekara, S. S. Williamson, and S. M. Lukic. Topological overview of hybrid electric and fuel cell vehicular power system architectures and configurations. IEEE Transactions on Vehicular Technology, 54(3):763-770, 2005.

L. Fernández, P. Garcia, C. Garcia, and F. Jurado. Hybrid electric system based on fuel cell and battery and integrating a single dc/dc converter for a tramway. Energy Conversion and Management, 52:2183-2192, 2011.

C. Guardiola, B. Pla, S. Onori, and G. Rizzoni. A new approach to optimally tune the control strategy for hybrid vehicles applications. In IFAC Workshop on Engine and Powertrain Control, Simulation and Modeling E-COSM'12, Rueil-malmaison, France, October 2012.

L. Guzzella and A. Sciarretta. Vehicle Propulsion Systems. Introduction to Modeling and Optimization. Springer-Verlag, Berlin, 2005.

T. Katrašnik. Hybridization of powertrain and downsizing of ic engine a way to reduce fuel consumption and pollutant emissions part 1. Energy Conversion and Management, 48:1411-1423, 2007a.

T. Katrašnik. Hybridization of powertrain and downsizing of ic engine analysis and parametric study part 2. Energy Conversion and Management, 48:1424-1424, 2007b.

S. Kermani, S. Delprat, T. Guerra, R. Trigui, and B. Jeanneret. Predictive energy management for hybrid vehicle. Control Engineering Practice, 20(4):408-420, 2012.

N. Kim, S. Cha, and H. Peng. Optimal control of hybrid electric vehicles based on pontryagins minimum principle. IEEE Transactions on Control Systems Technology, 19(5):1279-1287, 2011.

F. Lewis and V. Syrmos. Optimal Control. Wiley-Interscience, New York, 1995.

C. C. Lin, H. Peng, J. W. Grizzle, J. Liu, and M. Busdiecker. Control system development for an advanced-technology medium-duty hybrid electric truck. International Truck and Bus Meeting and Exhibition, 2003.

J. V. Mierlo, G. Maggetto, and P. Lataire. Which energy source for road transport in the future? a comparison of battery, hybrid and fuel cell vehicles. Energy Conversion and Management, 47:2748-2760, 2006.

F. Millo, L. Rolando, F. Mallamo, and R. Fuso. Development of an optimal strategy for the energy management of a range-extended electric vehicle with additional noise, vibration and harshness constraints. Proceedings of the Institution of Mechanical Engineers, Part D: Journal of Automobile Engineering, 227(1):4-16, 2013.

H. Mosbech. Optimal control of hybrid vehicle. In Proceedings of the International Symposium on Automotive Technology E Automation, pages 303-310, 1980.

S. J. Moura, H. K. Fathy, D. C. Callaway, and J. L. Stein. A stochastic optimal control approach for power management in plug-in hybrid electric vehicles. IEEE Transactions on Control Systems Technology, 19(3):545-555, 2011.

C. Musardo, G. Rizzoni, Y. Guezennec, and B. Staccia. A-ECMS: An adaptive algorithm for hybrid electric vehicle energy management. European Journal of Control, Fundamental Issues in Control, Special Issue, 11(4-5), 2005.

D. Naidu. Optimal Control Systems. CRC Press, 2003.

T. Nuesch, M. Wang, C. Voser, and L. Guzzella. Optimal energy management and sizing for hybrid electric vehicles considering transient emissions. In IFAC Workshop on Engine and Powertrain Control, Simulation and Modeling E-COSM'12, Rueil-malmaison, France, October 2012.

S. Onori, L. Serrao, and G. Rizzoni. Adaptive equivalent consumption minimization strategy for hybrid electric vehicles. In Proceedings of the 2010 ASME Dynamic Systems and Control Conference, 2010.

G. Paganelli, G. Ercole, A. Brahma, Y. G. Guezennec, and G. Rizzoni. General supervisory control policy for the energy optimization of chargesustaining hybrid electric vehicles. JSAE Review, 22(4):511-518, 2001.

L. Pontryagin, V. G. Boltyanskii, R. V. Gamkrelidze, and E. F. Mishchenko. The mathematical theory of optimal processes. Wiley, NY, 1962.

A. Poursamad and M. Montazeri. Design of genetic-fuzzy control strategy for parallel hybrid electric vehicles. Control Engineering Practice, 16 (7):861-873, 2008.

G. Rizzoni, L. Guzzella, and B. M. Baumann. Unified modeling of hybrid electric vehicle drivetrains. IEEE/ASME Transactions on Mechatronics, 4(3):246-257, 1999.

N. J. Schouten, M. A. Salman, and N. A. Kheir. Energy management strategies for parallel hybrid vehicles using fuzzy logic. Control Engineering Practice, 11(2):171-177, 2003. 
A. Sciarreta and L. Guzzella. Control of hybrid electric vehicles. IEEE Control System Magazine, 27(2):60-70, 2007.

A. Sciarretta, M. Back, and L. Guzzella. Optimal control of parallel hybrid electric vehicles. IEEE Transactions on Control Systems Technology, 12(3):352-363, 2004.

L. Serrao, S. Onori, and G. Rizzoni. ECMS as realization of Pontryagin's minumum principle for HEV control. In 2009 American Control Conference, pages 3964-3969, Saint Louis, USA, June 2009.

L. Serrao, S. Onori, and G. Rizzoni. A comparative analysis of energy management strategies for hybrid electric vehicles. Journal of Dynamic Systems, Measurement, and Control, 133:1-9, 2011.

S. Stockar, V. Marano, M. Canova, G. Rizzoni, and L. Guzzella. Energy-optimal control of plug-in hybrid electric vehicles for real-world driving cycles. IEEE Transactions on Vehicular Technology, 2011.

O. Sundström and L. Guzzella. A generic dynamic programming Matlab function. In 18th IEEE International Conference on Control Applications Part of 2009 IEEE Multi-conference on Systems and Control, pages 1625-1630, Saint Petersburg, Russia, July 2009.

O. Sundström, D. Ambühl, and L. Guzzella. On implementation of dynamic programming for optimal control problems with final state constraints. Oil E Gas Science and Technology Rev. IFP, 65(1):91-102, 2010.

T. van Keulen, D. van Mullem, B. de Jager, J. T. Kessels, and M. Steinbuch. Design, implementation, and experimental validation of optimal power split control for hybrid electric trucks. Control Engineering Practice, 20(5):547-558, 2012.

X. Wei, L. Guzzella, V. Utkin, and G. Rizzoni. Model-based fuel optimal control of hybrid electric vehicle using variable structure control systems. Journal of Dynamic Systems, Measurement and Control, Transactions of the ASME, 129(1):13-19, 2007. cited By (since 1996)24.

J. S. Won and R. Langari. Intelligent energy management agent for a parallel hybrid vehicle - part ii: torque distribution, charge sustenance strategies, and performance results. IEEE Trans. Vehicle Technol., 54(3):935-953, 2005.

W. Xiong, Y. Zhang, and C. Yin. Optimal energy management for a seriesparallel hybrid electric bus. Energy Conversion and Management, 50: 1730-1738, 2009. 